\title{
Cryogenic Ion Spectroscopy for Identification of Monosaccharide Anomers
}

\author{
Valeriu Scutelnic and Thomas R. Rizzo* \\ Laboratory of Molecular Physical Chemistry, École Polytechnique Fédérale de \\ Lausanne, Station 6, CH-1015 Lausanne, Switzerland. \\ *email address: thomas.rizzo@epfl.ch
}

\begin{abstract}
We combine conformer-selective, cryogenic infrared spectroscopy, quantum mechanical computations, and ${ }^{18} \mathrm{O}$ substitution at the reducing end to determine the structural preferences of protonated glucosamine in the gas phase. Cryogenic infrared-infrared (IR-IR) double resonance spectroscopy of helium-tagged protonated glucosamine provides vibrational fingerprints of individual conformers, and ${ }^{18} \mathrm{O}$ isotopic labeling facilitates the match with computed structures and provides a selective probe of the anomeric hydroxyl. This is key for using vibrational spectroscopy for glycan analysis and determining the generality of anomeric memory during glycosidic bond cleavage.
\end{abstract}




\section{Introduction}

Glycans are ubiquitous in biological systems, exhibiting a wide spectrum of functions. For example, they decorate the surface of cells, where they take part in cell-to-cell recognition, cellular signaling, and immune response via interactions with intrinsic and extrinsic glycanbinding proteins. ${ }^{1,2}$ Although mass spectrometry (MS) is one of the most sensitive techniques for the analysis of glycans, by itself it has difficulties in determining their primary structure because of their intrinsic isomeric complexity. ${ }^{3-5}$ Consequently, MS is often combined with other techniques for glycan analysis., ${ }^{6} 7$ One such technique, vibrational spectroscopy, has begun to show promise for identifying glycan isomers, owing to its extremely high sensitivity to specific hydrogen-bonding patterns. ${ }^{8,9}$ Polfer and coworkers first used infrared multiphoton dissociation (IRMPD) to measure vibrational spectra of gas-phase monosaccharides, ${ }^{10,11}$ and Schindler et al. extended this approach to determine glycosidic bond anomericity of small glycans. ${ }^{12,13}$ However, room-temperature spectral fingerprints of larger glycans often exhibit broad features that could render the structural identification ambiguous. ${ }^{14}$ While thermal broadening can be largely eliminated by cooling the samples to cryogenic temperature, ${ }^{8,9}$ spectral complexity can also arise from a multiplicity of stable conformers, each of which will have a different vibrational spectrum. In this case, conformer-selective spectroscopic techniques can often further simplify the spectrum. Voss et al. applied both cryogenic cooling and IR-IR double resonance spectroscopy to identify eight distinct conformers of sodiated glucose. ${ }^{15}$ Their results demonstrate that carbohydrates are prone to adopt multiple kineticallytrapped conformers in the gas phase. Their high spectral resolution allows them to unambiguously establish the anomeric configuration at the $\mathrm{C} 1$ carbon by comparing computed and experimental conformer-specific spectra. This may have important implications for glycan sequencing in light of the recent proposal by Schindler et al. that the anomeric memory of the glycosidic bond may be retained upon dissociation. ${ }^{12}$

Although isotopic labeling has proved to be an invaluable tool for the structural assignment of peptides, ${ }^{16-18}$ it has not been exploited for the study of glycans. In this work we combine IR-IR double-resonance spectroscopy in a cryogenic ion trap with ${ }^{18} \mathrm{O}$ labeling and quantum mechanical computations to determine the conformational preferences of a prototype system - protonated glucosamine. Glucosamine presents several advantages for testing and validating this new combination of techniques: (1) it protonates easily due to a basic amino group, (2) its compact size and rigid ring structure restrict the number of possible conformations, allowing a deeper understanding of the system, and (3) the presence of two types of oscillators ( $\mathrm{NH}$ and $\mathrm{OH}$ ) give rise to bands in two distinct regions of the spectrum that will allow us to distinguish them. 


\section{Experimental approach and computations}

A detailed description of the instrument in which we perform these experiments has been reported previously. ${ }^{19}$ Briefly, protonated glucosamine ions are produced by electrospraying a $0.1 \mathrm{mM}$ solution in a 50:50:0.1 methanol-water-acetic acid. The ions of a particular $\mathrm{m} / \mathrm{z}$ ratio are selected in a quadrupole mass filter and guided to a cryogenic octupole ion trap maintained at $3.5 \mathrm{~K}$. There they undergo collisions with helium buffer gas, which cools them sufficiently to allow the formation of weakly bound complexes of glucosamine with one or more helium atoms. The helium-tagged ions are then irradiated with an infrared (IR) pulse from a tunable optical parametric oscillator (OPO). When the OPO frequency is in resonance with a vibrational transition, IR radiation is absorbed and then redistributed, causing the weakly bound He atom to evaporate. Monitoring the depletion of the tagged ions as a function of the OPO wavenumber allows us to acquire an infrared absorption spectrum of all the conformers present. In order to obtain infrared spectra of individual conformers, two IR pulses are used in a double-resonance scheme. An IR pump pulse, firing at $5 \mathrm{~Hz}$, is fixed at a wavenumber corresponding to an absorption band of a specific conformer and is of sufficiently high fluence to saturate the transition. A probe IR pulse, firing at $10 \mathrm{~Hz}$ and arriving $1 \mu$ s after the pump pulse, is scanned in wavenumber, ${ }^{20,} 21$ inducing depletion when in resonance with any conformer that is not pumped. Taking the difference between the cycle with and without the pump pulse produces a dip spectrum of the pumped conformer.

Isotopic labeling with ${ }^{18} \mathrm{O}$ at the reducing end is achieved by dissolving $1.5 \mathrm{mg}$ of dry glucosamine hydrochloride (Sigma-Aldrich, 99\% purity) in $150 \mu \mathrm{L}$ of $\mathrm{H}_{2}{ }^{18} \mathrm{O}$ (Sigma-Aldrich, $97 \%$ purity) and maintaining the mixture at room temperature for 48 hours. The solution is diluted in $\mathrm{MeOH}(1: 1000)$ before electrospraying. ${ }^{22}$ It is known that only the $\mathrm{OH}$ at the reducing end exchanges under these conditions. ${ }^{23}$

To compute the most stable structures, we sample the conformational space using a

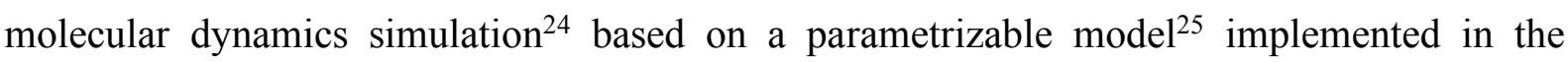
computer code PRIRODA. ${ }^{26}$ From such a simulation for the $\alpha$ anomer we collect 1001 geometries, which are then optimized, leading to the identification of 63 unique conformers. These unique geometries are further optimized by DFT using the B3LYP functional and a 6$31+\mathrm{G}(\mathrm{d}, \mathrm{p})$ basis set as implemented in Gaussian 09. ${ }^{27}$ The vibrational spectra of the lowestenergy conformers are computed with the same functional and basis set. The harmonic frequencies are uniformly scaled by a factor of 0.955 .

\section{Results and discussion}

In the range of $3150-3750 \mathrm{~cm}^{-1}, \mathrm{NH}$ and $\mathrm{OH}$ stretch bands are the most prominent. The infrared spectrum of protonated glucosamine, shown in Figure 1 (red trace), exhibits 13 distinct 
lines in this region - more than the total number of $\mathrm{NH}$ and $\mathrm{OH}$ oscillators in the molecule. This implies that multiple conformers are likely to be present. Among all the $\mathrm{NH}$ and $\mathrm{OH}$ oscillators, only the reducing end $\mathrm{OH}$ can be easily isotopically labeled by dissolving dry glucosamine in $\mathrm{H}_{2}{ }^{18} \mathrm{O}$. Isotopic substitution of ${ }^{18} \mathrm{O}$ causes only two bands to shift (blue trace in Figure 1), each by $12 \mathrm{~cm}^{-1}$, allowing us to assign these lines to anomeric $\mathrm{OH}$ vibrations. Assuming that a single line shifts for each conformer, this indicates the presence of at least two conformers coexisting in the gas phase.

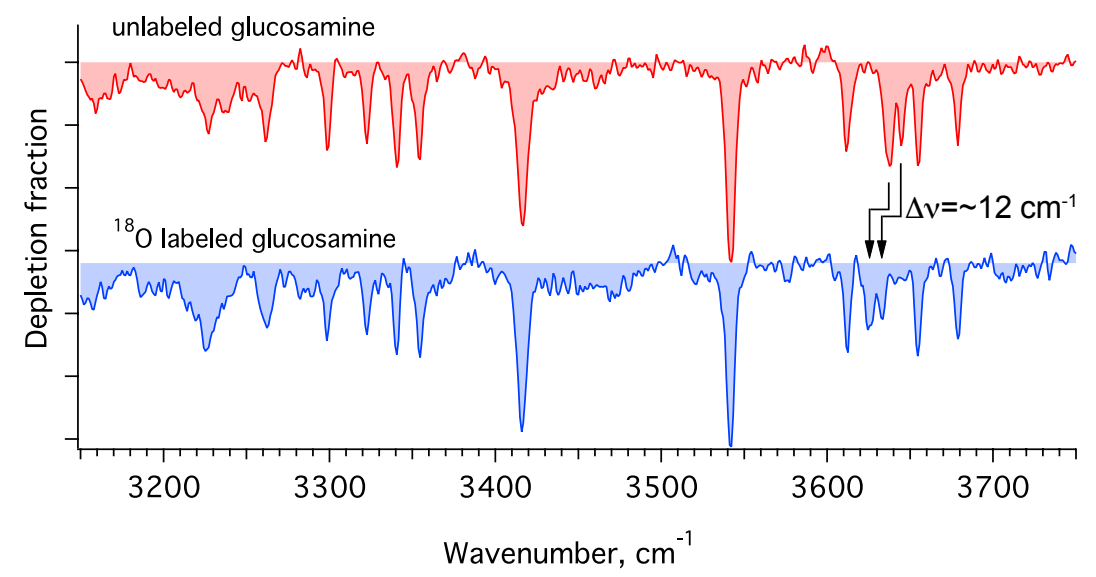

Figure 1. Infrared spectrum of glucosamine- $\mathrm{H}^{+}$(red) and glucosamine- $\mathrm{H}^{+}$labeled at the glycosidic $\mathrm{OH}$ with ${ }^{18} \mathrm{O}$ (blue).

The contribution of multiple conformers to the infrared spectrum renders its interpretation ambiguous. We thus applied IR-IR ion dip spectroscopy to acquire vibrational fingerprints of individual conformers (Figure 2). Vibrations of the $\mathrm{OH}$ group are expected to appear in the spectral region above $3400 \mathrm{~cm}^{-1},{ }^{15}$ and $\mathrm{NH}$ group vibrations usually appear lower than $3400 \mathrm{~cm}^{-1} .{ }^{28}$ Fixing the wavenumber of the IR pump pulse on an $\mathrm{OH}$ band at $3542 \mathrm{~cm}^{-1}$ and scanning the wavenumber of the probe pulse produces a spectrum with eight bands - more than the total number of stretch vibrations in the molecule - indicating that multiple conformers absorb at this wavenumber (Figure S4). However, pumping at $3417 \mathrm{~cm}^{-1}$ we obtain a spectrum consisting of five bands (see Figure 2), which we attribute to a single conformer (A). Fewer bands than the total number of stretch vibrations indicates that some are either strongly broadened or overlapping other bands. By fixing the IR pump pulse on an NH band at $3340 \mathrm{~cm}^{-}$ ${ }^{1}$ we obtain the fingerprint IR spectrum of a second conformer (B), and fixing it at $3323 \mathrm{~cm}^{-1}$ produces a third distinct spectrum (conformer C). The sum of conformer-specific spectra of A, $\mathrm{B}$ and $\mathrm{C}$ (Figure S5), reproduces well the single-laser spectrum, indicating that the most abundant conformers of glucosamine- $\mathrm{H}^{+}$are successfully sampled. The overlap of the anomeric $\mathrm{OH}$ bands in spectra $\mathrm{B}$ and $\mathrm{C}$ explains the shift of only two lines, rather than three, in the ${ }^{18} \mathrm{O}$ isotopic labeling experiment (Figure 1). 


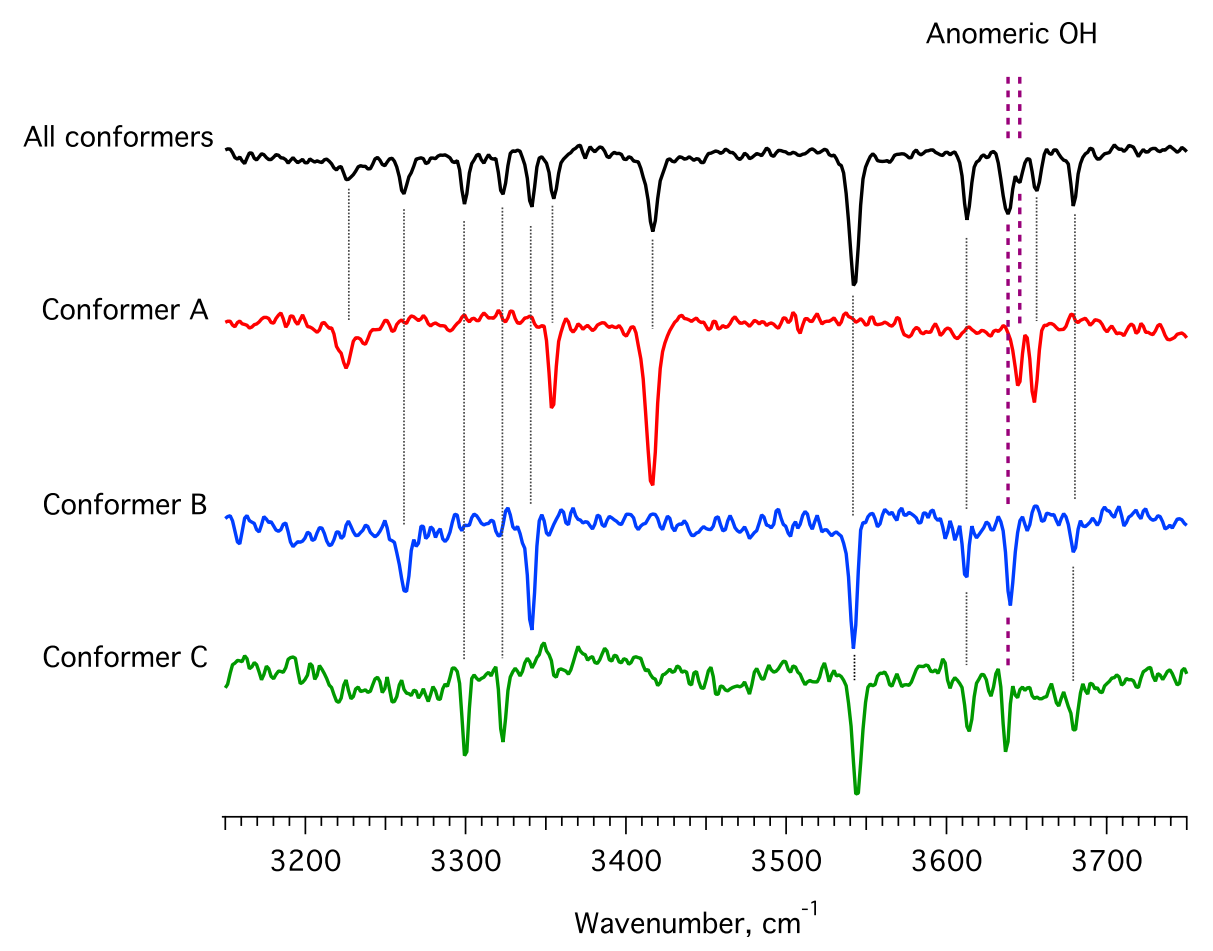

Figure 2. The top trace (black) is the non-conformer specific spectrum. The three spectra below are double resonance spectra of conformers A-C. The purple dashed lines indicate the bands assigned to anomeric hydroxyl by ${ }^{18} \mathrm{O}$ substitution (Figure 1).

The distinctive $\mathrm{OH}$ band pattern in the spectrum of conformer $\mathrm{A}$, as compared with $\mathrm{B}$ and $\mathrm{C}$, indicates a different hydrogen-bonding network in the former. Even though the positions of the $\mathrm{OH}$ bands are nearly identical in the spectra of conformers B and $\mathrm{C}$, the $\mathrm{NH}$ bands are highly sensitive to fine changes of their environment, allowing us to distinguish them. Considering the isotopic labeling (Figure 1) and the IR spectra of each conformer (Figure 2), we identify the bands of the anomeric $\mathrm{OH}$ groups: 3645,3640 and $3637 \mathrm{~cm}^{-1}$ (in spectra A, B and $\mathrm{C}$, respectively).

In order to determine the structures of the three conformers, we perform a conformational search using a molecular dynamics simulation at $1200 \mathrm{~K}$. Analyzing the generated conformations, we observed that the lowest in energy can be determined following a set of rules: 1) the pyranose cycle can adopt chair, boat or skewed-boat conformations (halfchair and envelope are usually transition-state geometries);29 2) the skewed-boat conformation is $>5 \mathrm{kcal} / \mathrm{mol}$ higher in energy than a chair conformation; 3 ) the group repulsion (in the case of large axial substituents in chair conformations) imposes an energetic penalty that can be compensated with efficient hydrogen bonds; 4) charge directs the hydrogen bonding pattern, ${ }^{30}$ and 5) hydrogen bonds that complete a ring of 6 or 7 atoms are stronger than 5-atom rings. The generated pool of conformers is further optimized at the B3LYP level of theory.

Figure 3 illustrates the comparison of the experimental and calculated spectra of the lowest-energy structures. Theoretical vibrational spectra and the computed structures that give 
rise to them generally agree well with the experimental spectra; moreover, they are consistent with the assignments of the reducing end $\mathrm{OH}$ bands (Figure 2). Our structures are not unlike the ones proposed by Schindler et al. in their study of glucosamine anomers with liquid chromatography and IRMPD. ${ }^{31}$ The computed structure for conformer A adopts a ${ }^{1} \mathrm{C}_{4}$ chair conformation, while for conformers $\mathrm{B}$ and $\mathrm{C}$ it has a ${ }^{4} \mathrm{C}_{1}$ configuration, and this explains their difference in the $\mathrm{OH}$ stretch region of the spectrum (see Figure 3). In agreement with Voss et al., ${ }^{15}$ we find the hydrogen-bond lengths being strongly correlated with the $\mathrm{OH}$ stretch frequency. Conformer $\mathrm{A}$ is lowest in energy due to strong hydrogen bonding (closing a 7membered cycle), which is reflected in the large shift of the $\mathrm{OH}$ band to $3417 \mathrm{~cm}^{-1}$; the corresponding hydrogen-bond length of this $\mathrm{OH}$ group is $1.8 \AA$ (Figure $3 \mathrm{a}$ ). In conformer $\mathrm{B}$, two $\mathrm{OH}$ groups form hydrogen bonds with lengths of 2.0 and $2.5 \AA$, their corresponding frequencies being 3542 and $3611 \mathrm{~cm}^{-1}$ respectively. The pattern of $\mathrm{OH}$ bands of conformer $\mathrm{C}$ strongly resembles that of conformer B, showing differences in positions of not more than 3 $\mathrm{cm}^{-1}$. The anomeric $\mathrm{OH}$ group serves as a hydrogen bond acceptor for the neighboring amino group, which makes the NH spectral region sensitive to structural changes between the $\alpha$ - and $\beta$-anomers. The anomeric $\mathrm{OH}$ bands of the conformers $\mathrm{B}$ and $\mathrm{C}$ occur at 3640 and $3637 \mathrm{~cm}^{-1}$ respectively; the difference reflecting a different hydrogen-bond strength with the NH group. The strong $\left(\sim 40 \mathrm{~cm}^{-1}\right)$ redshift of the anomeric $\mathrm{OH}$ bands from the highest resonant frequency of an alcohol $\mathrm{OH}$ group $\left(3680 \mathrm{~cm}^{-1}\right)$ can be explained by the inductive effect of the neighboring oxygen atom inside the pyranose ring, which weakens the reducing end $\mathrm{OH}$ bond force constant. 

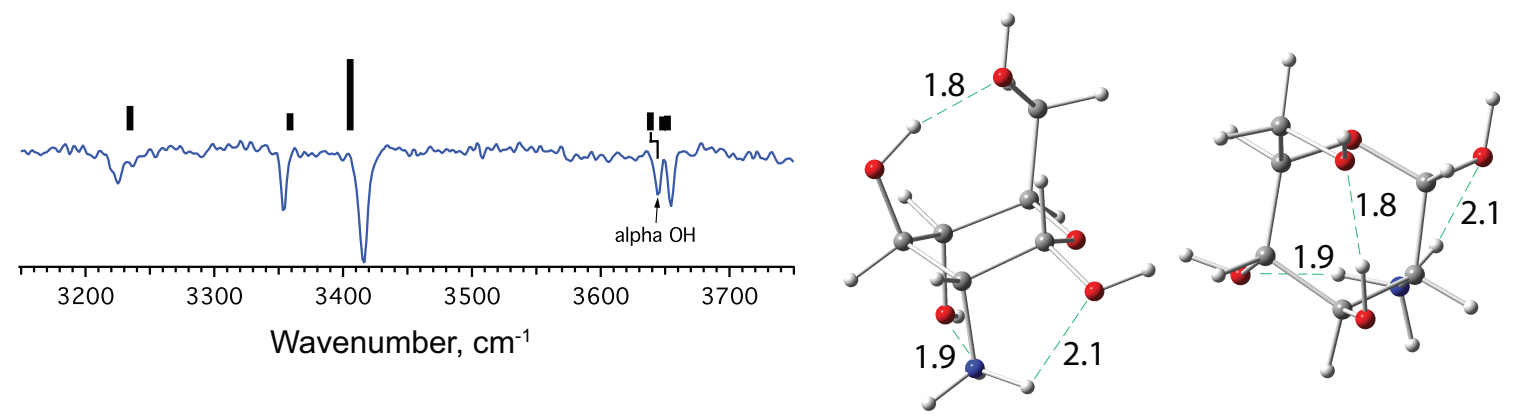

b) Conformer B

Chair ${ }^{4} \mathrm{C}_{1}, \alpha$-anomer

$(0.6 \mathrm{kcal} / \mathrm{mol})$
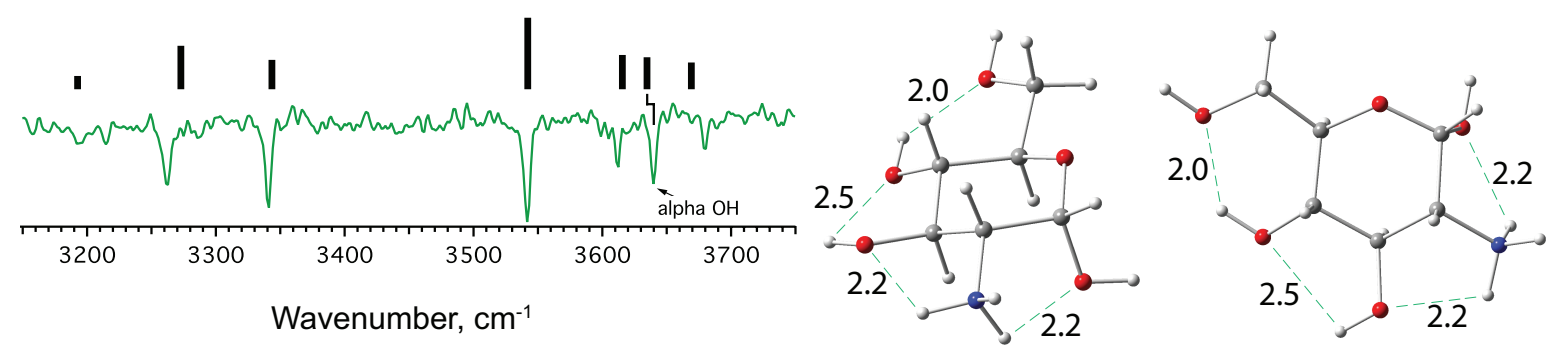

c) Conformer C
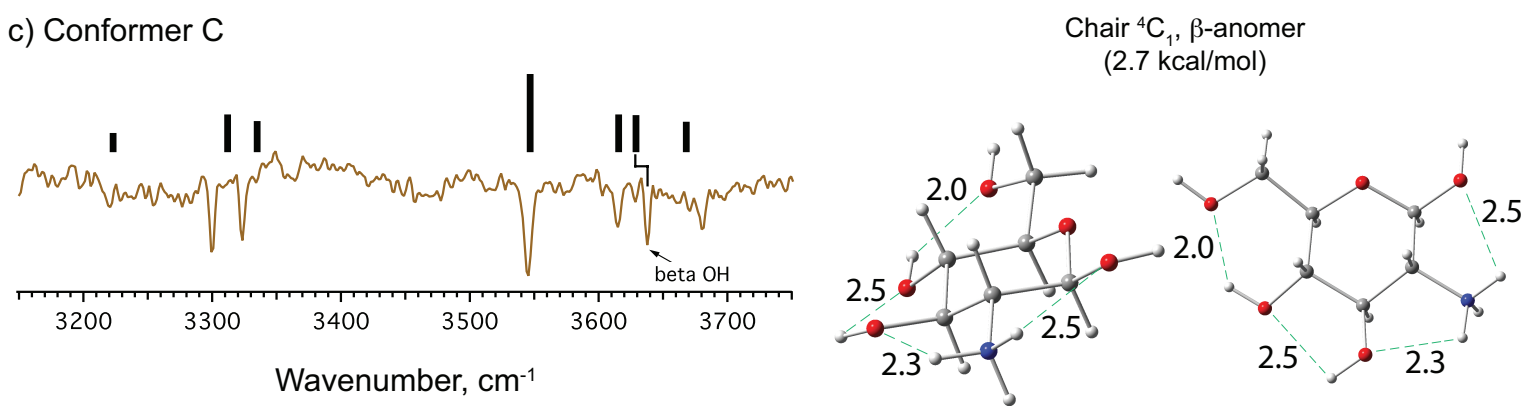

Figure 3. Comparison of computed spectra (black bars) with the measured spectra. The corresponding structure is shown to the right from two viewpoints. Relative energies including zero-point energy corrections are given in brackets. Hydrogen bonds are depicted with green dashes and their length is given in $\AA$.

Previous studies determined the $\alpha: \beta$ ratio of glucosamine in solution to be $1.7: 1$ (polarimetric measurements and $\mathrm{NMR}^{32}$ ) and 2.6:1 (microchip capillary electrophoresis ${ }^{33}$ ). In the gas phase, one can use the IR fluence dependence of the depletion to determine the relative abundance of the different conformers. We tune the IR OPO to a frequency where only one conformer absorbs and change the IR fluence, which is achieved by changing the pulse energy and keeping the photon beam area the same. Assuming that any He-tagged ion that absorbs an IR photon loses the tag, and extrapolating to infinite fluence, one obtains the approximate abundance of the probed conformer in the ion cloud. In this way, we estimate the relative population to be 0.19 for conformer A, 0.14 for conformer B, and 0.19 for conformer $\mathrm{C}$, or 
1.3:1:1.3 (see Figure S6). The $\alpha: \beta$ ratio of the gas-phase protonated glucosamine is thus $\sim 1.8: 1$, which is comparable to the solution-phase ratio. This is not surprising, since the large energy barrier does not allow the $\alpha / \beta$ mutarotation in the gas phase. However, conformers $A$ and $\mathrm{B}-$ both $\alpha$-anomers - are free to interchange in the gas phase, and the equal population of $\mathrm{A}$ and $\mathrm{B}$ found in the experiment is consistent with the small difference between their predicted free energies $(0.6 \mathrm{kcal} / \mathrm{mol}$ at $0 \mathrm{~K}$, which further decreases to $-0.1 \mathrm{kcal} / \mathrm{mol}$ at $300 \mathrm{~K})$.

In contrast to protonated glucosamine studied here, sodiated glucose exhibits a larger number of conformers due to strong binding of the coordinating metal to a few hydroxyl groups that hinders the interconversion of conformers. ${ }^{15,34}$ The presence of a proton in glucosamine does not hinder the interconversion of conformers as strongly as the coordinating metal.

\section{Conclusions}

Protonated glucosamine is analyzed by conformer-selective cryogenic infrared spectroscopy combined with quantum mechanical modeling and isotopic substitution of the reducing end. The well-resolved spectra provide critical insights into the nature of its hydrogenbonding interactions. Facile ${ }^{18} \mathrm{O}$ labeling of the reducing end combined with cryogenic IR spectroscopy allows us to estimate its conformational heterogeneity. By its ability to distinguish anomers, cryogenic ion spectroscopy and isotopic labeling hold promise for the characterization of collision-induced dissociation (CID) fragments of oligosaccharides, and, in particular, test the generality of anomeric memory during glycosidic bond cleavage. ${ }^{12}$

\section{Acknowledgements}

The authors thank the Swiss National Science Foundation (200020_165908) and the EPFL for their generous support of this work.

\section{Supporting Information for Publication}

Additional experimental details related to the determination of conformer populations. This information is available free of charge via the Internet at http://pubs.acs.org.

\section{References}

1. Varki, A., Biological Roles of Glycans. Glycobiology 2017, 27 (1), 3-49.

2. Rudd, P. M.; Elliott, T.; Cresswell, P.; Wilson, I. A.; Dwek, R. A., Glycosylation and the Immune System. Science 2001, 291 (5512), 2370-2376.

3. Hofmann, J.; Hahm, H. S.; Seeberger, P. H.; Pagel, K., Identification of Carbohydrate Anomers Using Ion Mobility-Mass Spectrometry. Nature 2015, 526, 241.

4. Kailemia, M. J.; Ruhaak, L. R.; Lebrilla, C. B.; Amster, I. J., Oligosaccharide Analysis by Mass Spectrometry: A Review of Recent Developments. Anal. Chem. 2014, 86 (1), 196212. 
5. Pagel, K.; Harvey, D. J., Ion Mobility-Mass Spectrometry of Complex Carbohydrates: Collision Cross Sections of Sodiated N-Linked Glycans. Anal. Chem. 2013, 85 (10), 51385145.

6. Manz, C.; Pagel, K., Glycan Analysis by Ion Mobility-Mass Spectrometry and Gas-Phase Spectroscopy. Current Opinion in Chemical Biology 2018, 42, 16-24.

7. Morrison, K. A.; Clowers, B. H., Contemporary Glycomic Approaches Using Ion Mobility-Mass Spectrometry. Current Opinion in Chemical Biology 2018, 42, 119-129.

8. Masellis, C.; Khanal, N.; Kamrath, M. Z.; Clemmer, D. E.; Rizzo, T. R., Cryogenic Vibrational Spectroscopy Provides Unique Fingerprints for Glycan Identification. J. Am. Soc. Mass Spectrom. 2017, 28 (10), 2217-2222.

9. Mucha, E.; González Flórez, A. I.; Marianski, M.; Thomas, D. A.; Hoffmann, W.; Struwe, W. B.; Hahm, H. S.; Gewinner, S.; Schöllkopf, W.; Seeberger, P. H., et al., Glycan Fingerprinting Via Cold-Ion Infrared Spectroscopy. Angew. Chem. Int. Ed. 2017, 56 (37), 11248-11251.

10. Contreras, C. S.; Polfer, N. C.; Oomens, J.; Steill, J. D.; Bendiak, B.; Eyler, J. R., On the Path to Glycan Conformer Identification: Gas-Phase Study of the Anomers of Methyl Glycosides of N-Acetyl-D-Glucosamine and N-Acetyl-D-Galactosamine. Int. J. Mass Spectrom. 2012, 330-332, 285-294.

11. Tan, Y.; Zhao, N.; Liu, J.; Li, P.; Stedwell, C. N.; Yu, L.; Polfer, N. C., Vibrational Signatures of Isomeric Lithiated N-Acetyl-D-Hexosamines by Gas-Phase Infrared Multiple-Photon Dissociation (Irmpd) Spectroscopy. J. Am. Soc. Mass Spectrom. 2017, 28 (3), 539-550.

12. Schindler, B.; Barnes, L.; Renois, G.; Gray, C.; Chambert, S.; Fort, S.; Flitsch, S.; Loison, C.; Allouche, A.-R.; Compagnon, I., Anomeric Memory of the Glycosidic Bond Upon Fragmentation and Its Consequences for Carbohydrate Sequencing. Nat. Commun. 2017, 8.

13. Gray, C. J.; Schindler, B.; Migas, L. G.; Pičmanová, M.; Allouche, A. R.; Green, A. P.; Mandal, S.; Motawia, M. S.; Sánchez-Pérez, R.; Bjarnholt, N., et al., Bottom-up Elucidation of Glycosidic Bond Stereochemistry. Anal. Chem. 2017, 89 (8), 4540-4549.

14. Renois-Predelus, G.; Schindler, B.; Compagnon, I., Analysis of Sulfate Patterns in Glycosaminoglycan Oligosaccharides by Msn Coupled to Infrared Ion Spectroscopy: The Case of Galnac4s and Galnac6s. J. Am. Soc. Mass Spectrom. 2018, 29 (6), 1242-1249.

15. Voss, J. M.; Kregel, S. J.; Fischer, K. C.; Garand, E., Ir-Ir Conformation Specific Spectroscopy of Na+(Glucose) Adducts. J. Am. Soc. Mass Spectrom. 2017.

16. Stearns, J. A.; Seaiby, C.; Boyarkin, O. V.; Rizzo, T. R., Spectroscopy and Conformational Preferences of Gas-Phase Helices. Phys. Chem. Chem. Phys. 2009, 11 (1), 125-132.

17. Voronina, L.; Masson, A.; Kamrath, M.; Schubert, F.; Clemmer, D.; Baldauf, C.; Rizzo, T., Conformations of Prolyl-Peptide Bonds in the Bradykinin 1-5 Fragment in Solution and in the Gas Phase. J. Am. Chem. Soc. 2016, 138 (29), 9224-9233.

18. Pereverzev, A. Y.; Boyarkin, O. V., Exploring the Relevance of Gas-Phase Structures to Biology: Cold Ion Spectroscopy of the Decapeptide Neurokinin A. Phys. Chem. Chem. Phys. 2017, 19 (5), 3468-3472.

19. Svendsen, A.; Lorenz, U. J.; Boyarkin, O. V.; Rizzo, T. R., A New Tandem Mass Spectrometer for Photofragment Spectroscopy of Cold, Gas-Phase Molecular Ions. Rev. Sci. Instrum. 2010, 81 (7), 073107.

20. Pereverzev, A. Y.; Cheng, X.; Nagornova, N. S.; Reese, D. L.; Steele, R. P.; Boyarkin, O. V., Vibrational Signatures of Conformer-Specific Intramolecular Interactions in Protonated Tryptophan. J. Phys. Chem. A 2016, 120 (28), 5598-5608. 
21. Pereverzev, A. Y.; Kopysov, V.; Boyarkin, O. V., High Susceptibility of Histidine to Charge Solvation Revealed by Cold Ion Spectroscopy. Angew. Chem. Int. Ed. 2017, 56 (49), 15639-15643.

22. Tan, Y.; Polfer, N. C., Linkage and Anomeric Differentiation in Trisaccharides by Sequential Fragmentation and Variable-Wavelength Infrared Photodissociation. J. Am. Soc. Mass Spectrom. 2015, 26 (2), 359-368.

23. Fang, T. T.; Bendiak, B., The Stereochemical Dependence of Unimolecular Dissociation of Monosaccharide-Glycolaldehyde Anions in the Gas Phase: A Basis for Assignment of the Stereochemistry and Anomeric Configuration of Monosaccharides in Oligosaccharides by Mass Spectrometry Via a Key Discriminatory Product Ion of Disaccharide Fragmentation, M/Z 221. J. Am. Chem. Soc. 2007, 129 (31), 9721-9736.

24. Laikov, D. N., A Reversible Numerical Integrator of the Isokinetic Equations of Motion. Theoretical Chemistry Accounts 2018, 137 (11), 140.

25. Laikov, D. N., A New Parametrizable Model of Molecular Electronic Structure. J. Chem. Phys. 2011, 135 (13), 134120.

26. Laikov, D. N.; Ustynyuk, Y. A., Priroda-04: A Quantum-Chemical Program Suite. New Possibilities in the Study of Molecular Systems with the Application of Parallel Computing. Russ. Chem. Bull. 2005, 54 (3), 820-826.

27. Frisch, M. J.; Trucks, G. W.; Schlegel, H. B.; Scuseria, G. E.; Robb, M. A.; Cheeseman, J. R.; Scalmani, G.; Barone, V.; Mennucci, B.; Petersson, G. A., et al., Gaussian 09 Revision D.01. Gaussian Inc. Wallingford CT 2009: 2009.

28. Stearns, J. A.; Mercier, S.; Seaiby, C.; Guidi, M.; Boyarkin, O. V.; Rizzo, T. R., Conformation-Specific Spectroscopy and Photodissociation of Cold, Protonated Tyrosine and Phenylalanine. J. Am. Chem. Soc. 2007, 129 (38), 11814-11820.

29. Mayes, H. B.; Broadbelt, L. J.; Beckham, G. T., How Sugars Pucker: Electronic Structure Calculations Map the Kinetic Landscape of Five Biologically Paramount Monosaccharides and Their Implications for Enzymatic Catalysis. J. Am. Chem. Soc. 2014, 136 (3), 10081022.

30. Scutelnic, V.; Perez, M. A. S.; Marianski, M.; Warnke, S.; Gregor, A.; Rothlisberger, U.; Bowers, M. T.; Baldauf, C.; von Helden, G.; Rizzo, T. R., et al., The Structure of the Protonated Serine Octamer. J. Am. Chem. Soc. 2018.

31. Schindler, B.; Laloy-Borgna, G.; Barnes, L.; Allouche, A.-R.; Bouju, E.; Dugas, V.; Demesmay, C.; Compagnon, I., Online Separation and Identification of Isomers Using Infrared Multiple Photon Dissociation Ion Spectroscopy Coupled to Liquid Chromatography: Application to the Analysis of Disaccharides Regio-Isomers and Monosaccharide Anomers. Anal. Chem. 2018, 90 (20), 11741-11745.

32. Horton, D.; Jewell, J. S.; Philips, K. D., Anomeric Equilibria in Derivatives of Amino Sugars. Some 2-Amino-2-Deoxy-D-Hexose Derivatives1-3. J. Org. Chem. 1966, 31 (12), $4022-4025$

33. Skelley, A. M.; Mathies, R. A., Rapid on-Column Analysis of Glucosamine and Its Mutarotation by Microchip Capillary Electrophoresis. J. Chromatogr. A 2006, 1132 (1), 304-309.

34. Heaton, A. L.; Armentrout, P. B., Experimental and Theoretical Studies of Sodium Cation Interactions with D-Arabinose, Xylose, Glucose, and Galactose. J. Phys. Chem. A 2008, 112 (41), 10156-10167. 


\section{TOC Graphic:}

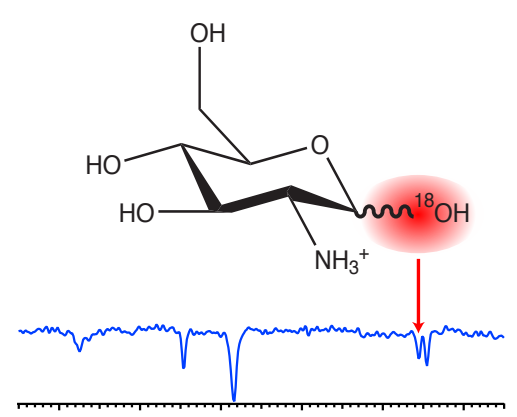

IR wavenumber 